\title{
Parallel LSTM-Based Regional Integrated Energy System Multienergy Source-Load Information Interactive Energy Prediction
}

\author{
Bo Wang $\left(\mathbb{D},{ }^{1}\right.$ Liming Zhang, ${ }^{1}$ Hengrui $\mathrm{Ma}^{2}$ Hongxia Wang $\mathbb{D},{ }^{1}$ and Shaohua Wan $\mathbb{D}^{3}$ \\ ${ }^{1}$ School of Electrical Engineering, Wuhan University, Wuhan 430070, China \\ ${ }^{2}$ Tus-Institute for Renewable Energy, Qinghai University, Xining, China \\ ${ }^{3}$ School of Information and Safety Engineering, Zhongnan University of Economics and Law, Wuhan 430073, China
}

Correspondence should be addressed to Shaohua Wan; shaohua.wan@ieee.org

Received 16 July 2019; Accepted 17 October 2019; Published 22 November 2019

Guest Editor: Xiaoqing Bai

Copyright (c) 2019 Bo Wang et al. This is an open access article distributed under the Creative Commons Attribution License, which permits unrestricted use, distribution, and reproduction in any medium, provided the original work is properly cited.

The multienergy interaction characteristic of regional integrated energy systems can greatly improve the efficiency of energy utilization. This paper proposes an energy prediction strategy for multienergy information interaction in regional integrated energy systems from the perspective of horizontal interaction and vertical interaction. Firstly, the multienergy information coupling correlation of the regional integrated energy system is analyzed, and the horizontal interaction and vertical interaction mode are proposed. Then, based on the long short-term memory depth neural network time series prediction, parallel long shortterm memory multitask learning model is established to achieve horizontal interaction among multienergy systems and based on user-driven behavioral data to achieve vertical interaction between source and load. Finally, uncertain resources composed of wind power, photovoltaic, and various loads on both sides of source and load integrated energy prediction are achieved. The simulation results of the measured data show that the interactive parallel prediction method proposed in this article can effectively improve the prediction effect of each subtask.

\section{Introduction}

With the depletion of fossil energy, the contradiction between energy demand growth and energy shortage in the process of social and economic development, energy utilization, and environmental protection is becoming more and more serious. Under such background, the consumption system of traditional energy production with fossil energy as the core has been difficult to sustain, so building a multienergy complementary and optimized energy supply and demand system and guiding the overall transformation of the energy industry have become the top priority of China's energy sector development [1]. On the basis of energy system source-network-load-storage vertical optimization, the multienergy supply systems are coordinated and optimized in a horizontal direction by the multienergy coupling relationships to realize integrated energy system for energy cascade utilization and multienergy coordinated scheduling, which will play a key role in the abovementioned energy revolution [2]. On the one hand, it will improve energy efficiency through comprehensive development and utilization of energy, and on the other hand, it will increase the potential energy penetration rate by converting electricity into heat, cold, natural gas, electric vehicle energy storage, etc.

The extension of IES in end users is called regional integrated energy system (RIES). In the RIES with intermittent renewable energy such as wind power/photovoltaic, due to the coupling and interconnection of various energy subsystems such as power system, thermal system, natural gas system, and transportation system, the energy consumption data are scattered and multidimensional of the versatile load such as electric load, heat load, and gas load on the user side. Such huge amount of data information is of great value in situational awareness, especially in new energy generation and load forecasting [3]. Zhang et al. [4] used 
data analysis and prediction algorithms to verify the correlation between energy consumption behavior and distributed PV output and input the prediction results into the predicted energy management system to optimize load-side energy consumption and balance the system's energy supply and load demand. Deng et al. [5] proposed the energy 5.0 concept based on cyber-physical-social systems (CPSS) and analyzed the social behavior characteristics of load-side energy consumption in smart home energy systems, by collecting a variety of operational information in the actual system by using the "data driven + physical model" approach to form a new energy forecasting agent to achieve optimal operational control [6-8]. In addition, in the energy prediction of RIES, deep learning prediction technology has attracted the attention of many scholars at home and abroad. Wang et al. [9] have made extensive analysis and discussion on renewable energy forecasting methods based on deep learning, discussed the current problems facing this direction, and prospected the future development direction. Wang et al. [10] proposed a wind speed prediction model based on deep confidence network and achieved good prediction results. Chen et al. [11] proposed a new two-layer nonlinear combination short-term wind speed prediction method (EEL-ELM). In the first layer, the extreme learning machine (ELM), Elman neural network (ENN), and long short-term memory (LSTM) neural network are used to predict wind speed, and three prediction results are obtained. Then, the nonlinear aggregation mechanism of extreme learning machine (ELM) is used to alleviate the inherent weakness of single method and linear combination. Liu et al. [12] combined variational mode decomposition (VMD), singular spectrum analysis (SSA), long short-term memory (LSTM) network, and extreme learning machine (LEM) and proposed a new multistep wind speed prediction model. The simulation results show that the method is more effective and robust in extracting trend information. On the basis of multiple long short-term memory (LSTM) learning, the literature [13] combines the extreme value optimization algorithm and the support vector machine model to integrate the LSTM layer prediction results and predicts the wind speed in a short time. In literature [14], the LSTM deep neural network algorithm is used, and the user's energy data of various types of equipment are used to predict the residential load on the load side in a short term, which proves the data value of the load energy information in the forecasting field. Multitask learning has been studied for about 20 years [15-17]. At present, multitask learning methods can be roughly summarized into two categories. One is to share the same parameters between different tasks, and the other is to mine the shared data features hidden between different tasks [18]. Argyriou et al. [19] detailed the four multitasking learning methods of feature selection, kernel selection, adaptive pooling, and graphical model structure. Jebara [20] proposed a typical multitask model for mining common features between multitasks. A framework for multitask feature learning is given in this paper, which becomes the basis for many multitask learning references [21, 22]. Inspired by the above literature, this paper argues that it is necessary to explore the value of multienergy coupling information and load-side user behavior characteristics for energy system operation and use the information correlation characteristics between multiple energy sources to expand the concept of multienergy interaction to the forecasting stage. The "information interaction" strategy is proposed and explored the data information implied in each link of RIES by combining deep learning technology, and the energy utilization efficiency is maximized through multienergy interaction. Based on the existing research, this paper comprehensively considers the horizontal and vertical information interaction characteristics between multiple energy loads in RIES and proposes a parallel energy LSTM based on multienergy-load information interactive shortterm energy prediction strategy. Firstly, the RIES multienergy-charge coupling correlation is analyzed, and the horizontal and vertical "information interaction" modes of multienergy load are proposed. Then, from the perspective of information interaction, based on the LSTM deep neural network time series prediction, the parallel LSTM multitask learning model is established to realize the horizontal interaction between multienergy systems. At the same time, based on the user's energy behavior data driving, the vertical interaction between the source and the load is realized, and the uncertain sources are used for integrated energy prediction of the wind power, photovoltaic, and various loads on both sides of the source and the load. Finally, based on the measured data of a region's RIES, the prediction model is trained and verified. The simulation results show that the proposed information interactive parallel prediction method can effectively improve the prediction effect of each subtask.

\section{Regional Integrated Energy System}

2.1. Basic Structure of Regional Integrated Energy System. The regional integrated energy system takes the electricity as the core, is based on smart grid, and is led by clean energy. It uses advanced information communication and energy conversion technologies to organically connect the links of producing, transporting, storing, and consuming in the multienergy systems such as electricity, gas, and heat so as to achieve optimal allocation of energy, multienergy coupling, and complement [23]. RIES structurally includes regional energy production input network, multienergy coupling network, and regional internal load-side energy output network. It can fully absorb all kinds of renewable energy such as wind power and photovoltaic and access various types of loads such as electricity, gas, heat, and cold to achieve efficient energy allocation. At the same time, its top layer has an integrated information communication support system to achieve the interactive fusion of energy flow and information flow. The basic architecture of the RIES described in this paper is shown in Figure 1.

\subsection{Characteristics of Regional Integrated Energy System} "Information Interconnection". Under the support of the energy Internet and multienergy key technologies characterized by "open" and "interconnected," the coupling interaction of multienergy-network-load-storage in RIES is 


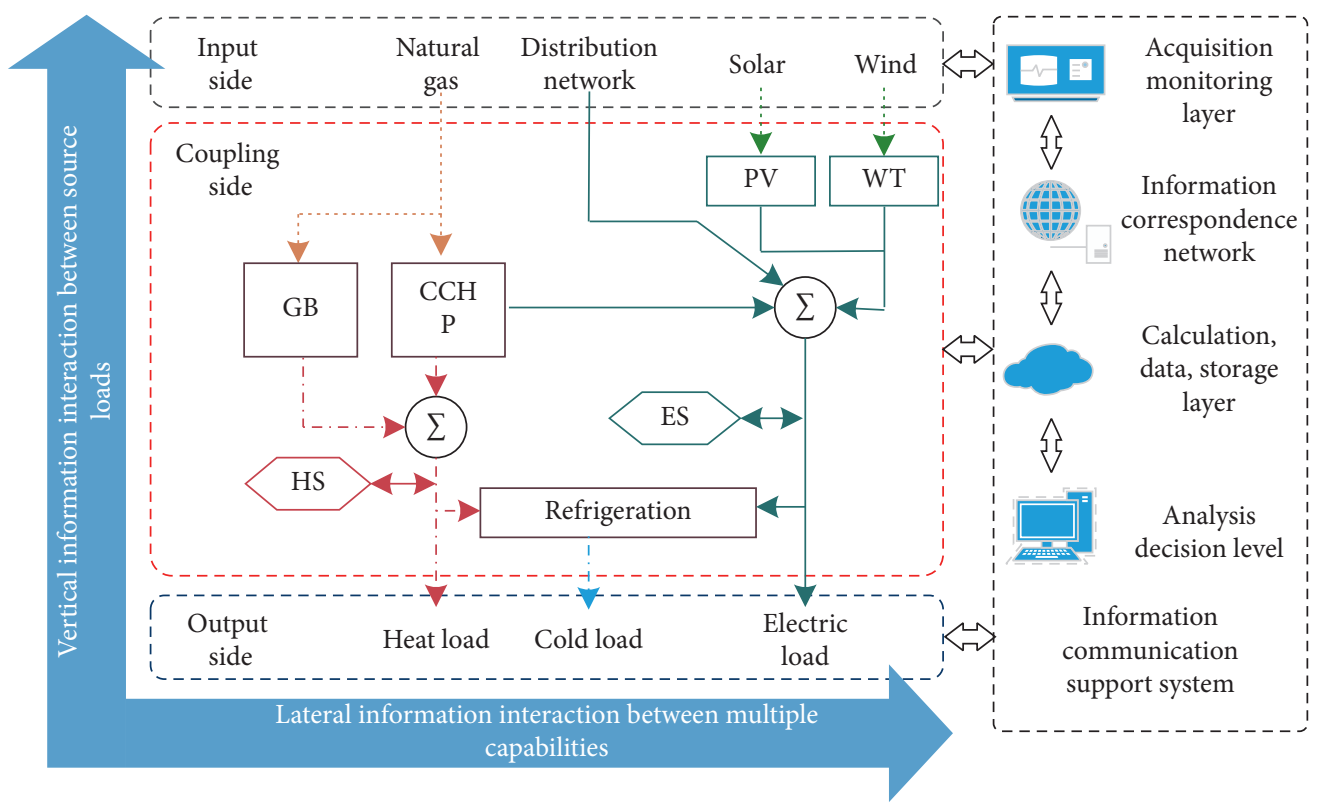

Figure 1: Basic framework of RIES.

more tight [24]. (1) From the perspective of horizontal interconnection, RIES will integrate the energy subsystems such as cold/heat/electric/gas/traffic to construct a multienergy flow system. The energy is converted and flowed between the systems through the coupling components, and the various energy forms complement each other. The energy resources have to be optimally configured in a wide area, that is, to achieve the "horizontal information interconnection" of the energy Internet. (2) From the perspective of vertical interconnection, based on the traditional energy supply network, RIES will realize the real-time sharing of information resources in the sourcenetwork-load-storage through Internet technology. This resource sharing mechanism has two-way conduction characteristics. The user has information bidirectional conduction capability between the information control system, the energy supply module, and the multienergy intelligent delivery module. The interactive sharing of energy information can also be realized between users, that is, to realize the "vertical information interconnection" of the energy Internet.

\section{RIES Multienergy Coupling Correlation Analysis}

3.1. RIES Horizontal Multienergy Information Coupling. The horizontal multienergy information coupling correlation of RIES is represented by the correlation between different energy flow subsystems such as electricity, gas, and heat.

From the perspective of coupling, the composition of the RIES can be divided into a coupling unit and a noncoupling unit. As shown in Figure 2, the coupling unit connects different energy flow subsystems and consumes one or more energy sources to generate other energy sources. Typical coupling forms are as follows: cold and heat electricity

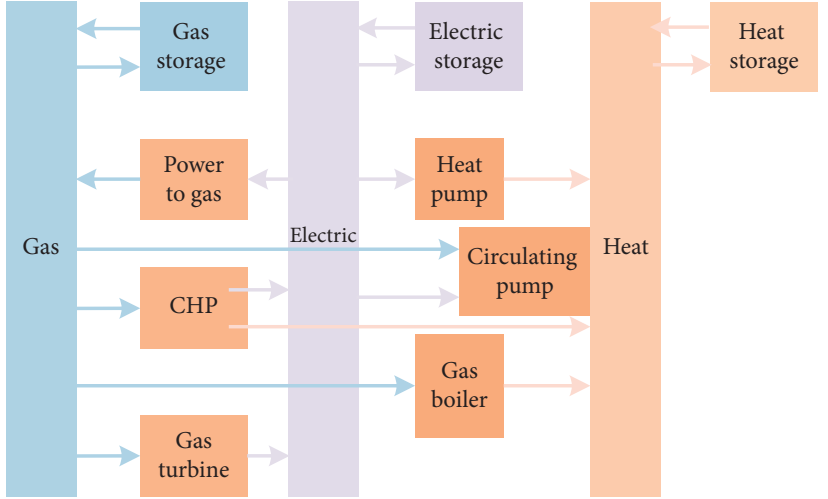

FIgURE 2: Transverse multicoupling schematic diagram.

supply units consume coal or natural gas to generate electricity, heat, and cold. The heat pump consumes electrical energy to generate thermal energy, the electrical hydrogen consumes electrical energy to generate hydrogen, and the coupling unit causes the energy flow subsystems to interact. The uncoupled unit includes the network and equipment inside each energy flow subsystem and other uncoupled boundary conditions such as source and load. The uncoupled unit also affects other systems through the coupling unit [25]. Therefore, different energy flow subsystems have strong coupling between them, and there is a large amount of horizontal multienergy coupling information existing in the multienergy system data, resulting in great correlation between various units within the system.

3.2. RIES Vertical Source-Load Information Coupling. The vertical source-load information coupling correlation of RIES is represented by the correlation between the multienergy supply of the source and the user's energy behavior of 
the load. Different energy behaviors correspond to different load characteristics [26]. For example, the lighting behavior in RIES is a typical user energy behavior. Through advanced measurement devices, the user's lighting behavior can be reflected as a lighting load curve.

From the perspective of user energy behavior, there are multiple coupling relationships between multiple energy loads in RIES. Taking the user's lighting behavior as an example, if the cloud suddenly covers a certain area and reduces the ambient light intensity, it will affect the user's lighting behavior: people will increase the lighting equipment usage to meet the lighting demand, and the lighting load will increase. At the same time, such cloud movements will also have an impact on photovoltaic power generation: cloud clusters will block the photovoltaic panels, which will affect the irradiance reduction on the photovoltaic panels and affect the photovoltaic power generation. In order to prove the characteristics of this behavioral feature, this paper takes the data of three days from January 24 to 26, 2016, of RIES in a certain area of Tianjin for quantitative analysis. The photovoltaic power output curve and the user lighting load curve are shown in Figure 3.

The upper part of Figure 3 is the curve trend of the photovoltaic power output, and the lower part is the curve trend of the user's lighting load. (1) Both the user's lighting load and the photovoltaic power output have a day and night cycle. The distribution of them is mainly concentrated in the daytime. The peak of the user's lighting load is generally distributed between 18:00 and 20:00, and the peak output of the photovoltaic power supply is generally distributed between 12:00 and 14:00 at noon. (2) Taking the three-day short-term data for comparison analysis, the overall output of photovoltaic power generation on the third day was significantly larger than the previous two days. Accordingly, the lighting load on the third day between 10:00 and 16:00 is reduced compared to the previous two days and it is reduced to the low of the day. It shows that when the output of photovoltaic power generation increases, it also affects the user's energy consumption behavior when the ambient light intensity is large, which means that the user's demand for lighting energy is reduced. (3) Take the ultra-short-term data analysis from 12:00 to $16: 00$ on the first day, and the photovoltaic power output reaches the peak of one day at $13: 00$, and the lighting load is also reduced to low of the daytime at this moment. During the period from 13:00 to $14: 00$, a sudden downward climb occurs, and the lighting load increases at the corresponding time. This "mutation" phenomenon should be caused by the cloudy weather affected by the cloud. From the above analysis, it can be found that there is a strong information coupling correlation between the "source" photovoltaic power output and the "charge end" user's lighting load.

3.3. RIES Multienergy Information Interaction Mode. RIES has both horizontal multienergy information coupling correlation characteristics and vertical source-load information coupling correlation characteristics. From the perspective of information interaction energy management,

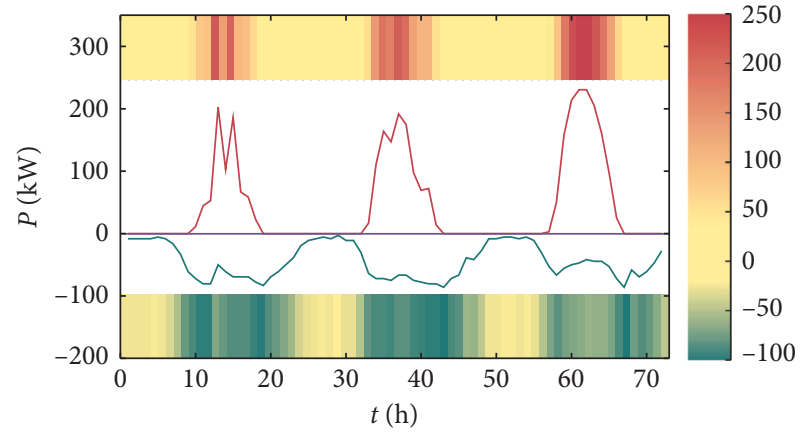

FIGURE 3: The relationship between the photovoltaic power curve and the lighting load curve of the user.

there are also two horizontal and vertical information interaction modes.

The horizontal information interaction mode is based on multienergy conversion information sharing. On the basis of obtaining the measurement data of the multienergy flow subsystem, the nonlinear artificial intelligence deep learning technology is used to process the multienergy data in parallel, and it effectively utilizes the complex shared information of energy conversion, identifies the abstract features of the training data, and improves the precision of subtask prediction of the energy management.

The vertical information interaction mode is based on user behavior information sharing. On the basis of obtaining multicategory data on both sides of the source and the load, the deep learning method is used to dig the user-side behavior characteristics, and the associated user behavior information data are taken as input to guide the source-side energy management prediction and improve the prediction accuracy of the energy supply end.

\section{Multitask Learning Mechanism for Deep Learning Energy Prediction}

\subsection{LSTM Recurrent Neural Network}

4.1.1. Principle of LSTM Cyclic Neural Network. The training objects of the source-charged uncertain resources have obvious timing characteristics, and the sequence data have strong correlation before and after. From the perspective of learning and training, recurrent neural network (RNN) can process sequences with temporal correlation of arbitrary length by using neurons with self-feedback, which has obvious advantages in processing sequence data [27]. The structure of the RNN is shown in Figure 4(a). The LSTM network is a variant of RNN that overcomes the traditional RNN gradient demise problem [28]. The hidden layer is no longer a simple neural unit, but an LSTM unit with a unique memory mode. Each LSTM unit contains a state cell that describes the current state of the LSTM unit [29]. $c$ represents the current state quantity of the LSTM unit, and $h$ represents the current output of the LSTM unit.

LSTM reads and modifies state units by controlling the forget gates, input gates, and output gates [30], which are 


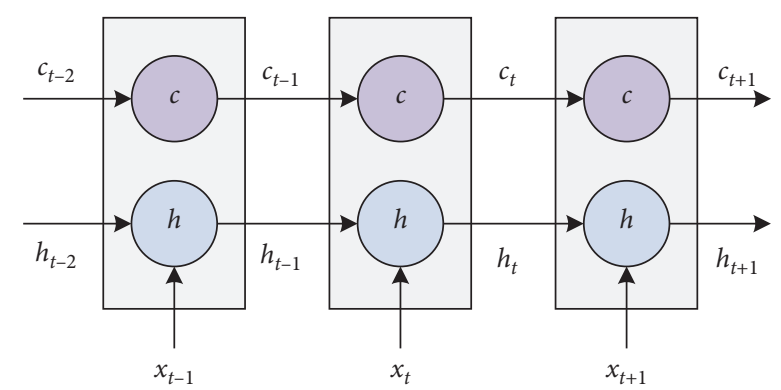

(a)

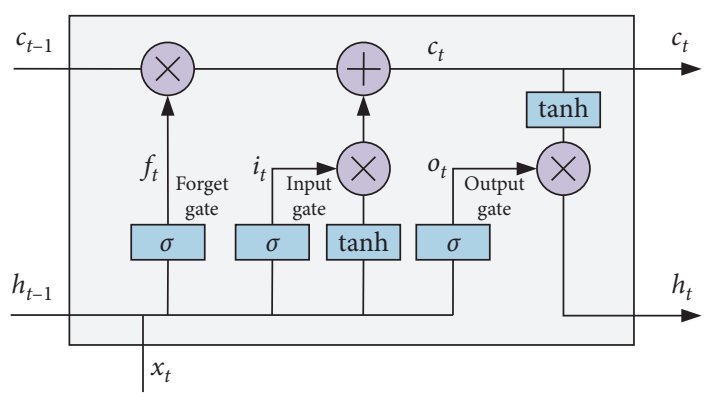

(b)

FIgURE 4: Schematic diagram of RNN and LSTM. (a) The structure of the RNN. (b) The structure of the LSTM cell.

typically formed by sigmoid or tanh functions and Hadamard product operation. As shown in Figure 4(b), the forget gate determines $c_{t-1}$ of the previous moment and how many components of the cell state $c_{t}$ remain at the current time, and the input gate determines how many components of the network input are saved to the cell state $c_{t}$ at the current time $x_{t}$ and how many components of the output gate control cell state $c_{t}$ are output to the current value $h_{t}$ of the LSTM.

The calculation formula between the variables of the LSTM unit is as follows:

$$
\begin{aligned}
f_{t} & =\sigma\left(W_{f x} x_{i}+W_{f h} h_{t-1}+b_{f}\right), \\
i_{t} & =\sigma\left(W_{i x} x_{i}+W_{i h} h_{t-1}+b_{i}\right), \\
g_{t} & =\phi\left(W_{g x} x_{t}+W_{g h} h_{t-1}+b_{g}\right), \\
c_{t} & =f_{t} \odot c_{t-1}+i_{t} \odot g_{t}, \\
o_{t} & =\sigma\left(W_{o x} x_{t}+W_{o h} h_{t-1}+b_{o}\right), \\
h_{t} & =o_{t} \odot \phi\left(c_{t}\right) .
\end{aligned}
$$

In the formula, $W_{f x}, W_{f h}, W_{i x}, W_{i h}, W_{g x}, W_{g h}, W_{o x}$, and $W_{\text {oh }}$ are the weight matrices corresponding to the input of the network activation function; $b_{f}, b_{i}, b_{g}$, and $b_{o}$ are the offset vectors; $\odot$ represents the multiplication of the Hadamard product matrix; $\sigma$ represents the sigmoid activation function, and $\phi$ represents the tanh activation function.

4.1.2. LSTM Deep Learning Training Algorithm. The training of LSTM network adopts BP-based backpropagation through time (BPTT) [31]. The algorithm steps are as follows: (1) Calculate the output value of each neuron forward. For the LSTM unit, the values of the six vectors, such as $f_{t}, i_{t}$, $g_{t}, c_{t}, o_{t}$, and $h_{t}$, are calculated. (2) Calculate the error term of each neuron in reverse. Similar to traditional RNN, the backpropagation of the LSTM error term consists of two levels: one is at the spatial level, and the error term is propagated to the upper layer of the network; the other is at the time level, which propagates back in time. For example, from the current time of $t$, the error at each moment is calculated. (3) Calculate the gradient of each weight according to the corresponding error term, and update the network weight parameter. (4) Jump to the first step and continue to perform the first 3 steps until the network error is less than the given value.
4.2. LSTM Network Multitask Learning Mechanism. Multitask learning (MTL), as an inductive migration mechanism, avoids the underfitting of model training by using the correlation between multiple tasks, digging the hidden common features, and improving the generalization learning ability of the model [32]. RIES source wind and light prediction and load-end electricity, gas, heat, and cold load prediction can be regarded as different tasks in multitask learning [33]. Multiple tasks share some common data or model structures, that is, there is a certain correlation between the multienergy prediction tasks. The multitask learning prediction model based on the LSTM network proposed in this paper shares the hidden layer nodes among all prediction tasks through the hard parameter sharing method and trains the training data corresponding to the multienergy prediction tasks at the same time, considering the correlation information between tasks to improve the learning ability of all predictive tasks [34]. A comparison of single-task and multitask learning based on the LSTM network is shown in Figure 5. The multisource historical data in the figure is a collection of five types of historical data including wind power, photovoltaic, electric load, heat load, and cold load.

\section{Parallel LSTM-Based RIES Information Interactive Energy Prediction Method}

5.1. RIES Multienergy Prediction Model Design. RIES has uncertainties at both the source and the load end, so accurate prediction of uncertain resources is the basis for implementing RIES energy optimization management and scheduling [35]. The source mainly includes wind power prediction and photovoltaic power prediction, and the load mainly includes electric load prediction, thermal load prediction, and cold load prediction.

As described in the second section of this paper, there are intricate mutual coupling relationships between multiple energy loads of RIES, and the prediction tasks of uncertain resources on both sides of the source and load have strong correlation. It can effectively improve the overall prediction accuracy of the RIES uncertain resources by learning this coupling relationship through joint prediction training [36]. From the perspective of RIES multienergy-load information interaction, this paper proposes the concept of "parallel 


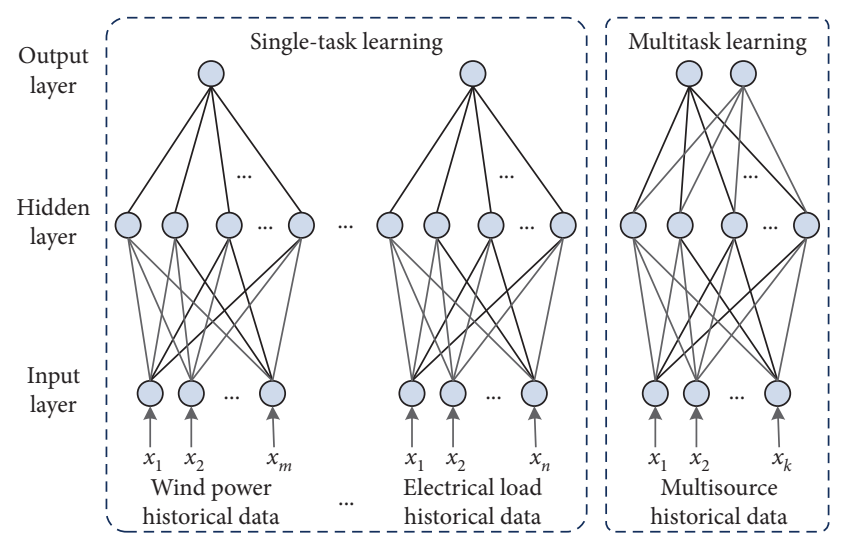

FIGURE 5: Comparison of single-task and multitasking learning based on the LSTM network.

prediction" and utilizes the strong nonlinear learning ability and historical memory advantages of LSTM deep learning network to conduct integrated prediction of uncertain resources at both source and load. As shown in Figure 6, the horizontal information interaction based on multienergy conversion information sharing mainly utilizes the multitask learning parameter sharing mechanism of the parallel LSTM prediction network; the vertical information interaction based on the user behavior information sharing mainly uses the user behavior data to guide source-load multitask prediction as an additional unified input information. Parametric sharing is weight sharing, which takes advantage of the correlation between subtasks. Input sharing refers to data sharing. Different subtasks share historical power data such as wind power, photovoltaic power, power load, thermal load, and cooling load.

5.2. Data Preprocessing. The training samples select the wind power generation time series on the source side, the photovoltaic power generation time series, the charge side on the load side, the heat load, the cold load time series, and the illumination load series data. Considering the input and output range of the nonlinear activation function in the model, in order to avoid neuron saturation, the data are normalized and mapped between $[-1,1]$, as shown in the following equation:

$$
x^{\prime}=\frac{x-\left(x_{\max }+x_{\min }\right) / 2}{\left(x_{\max }-x_{\min }\right) / 2} .
$$

In the equation, $x$ is the actual input or output data and $x_{\max }$ and $x_{\min }$ are the maximum and minimum values of the variable, respectively.

5.3. Task Construction. The essence of using the multitask learning method for multienergy-load integration prediction lies in the close correlation of each prediction subtask [37]. The traditional single-task prediction method uses a single dimension of historical observations to predict its future output, and the model output is a single attribute. The multitask learning integration prediction uses the multidimensional data on both sides of the source and the load as a common input to effectively utilize the sample. On the other hand, through the shared hidden layer node division method, the five attributes of wind, photovoltaic power generation, electricity, heat, and cold load are simultaneously predicted to play the role of inductive bias and improve prediction accuracy.

5.4. Model Training. The LSTM-based source-charge integrated prediction model mainly needs to determine the following parameters: input layer dimension, input layer time step, hidden layer number, each hidden layer dimension, and output layer dimension [38].

The time step of the input layer is generally the length of the variable time series used for the source-load integration prediction. According to the periodic characteristics of the prediction object sequence, the input layer time step is set to 24 , that is, the historical data whose resolution ratio is $1 \mathrm{~h}$ during the first seven days before the input is used for forecasting and it takes up one input node per day. The input layer dimension is the number of nodes. The input layer node of this paper contains six objects: wind power generation time series, photovoltaic power generation time series, charge side load, heat load, cold load time series, and lighting load series, which constitutes totally 42 nodes, so the input layer dimension is set to 42 . Since this paper simultaneously predicts six attributes of wind power, photovoltaic, electricity, heat, and cold load, the output layer has 5 nodes, that is, the output layer dimension is set to 5 . In this paper, based on experience and multiple trial and comparison, the number of hidden layers is set to 2 , and the dimension of the hidden layer is set to 30 . The selection of the activation function is as shown in Figure 4(a), the batch_size is set to 42 , the numbers of iterations of an epoch is set to 8784 , the learning rate is set to 0.80 , and the learning delay rate is set to 0.05 . The network training function uses the BPTT algorithm mentioned above.

5.5. Evaluation Indicators. Multienergy-load integration forecasting simultaneously trains multiple forecasting tasks. To comprehensively measure the forecasting effect, it is necessary to evaluate the integrated forecasting accuracy as a whole [39]. In this paper, the average accuracy evaluation index based on weight coefficient is proposed. For the importance degree of different energy on the two sides of the RIES, the corresponding weight coefficient is given to different energy output types. For an RIES, the uncertainties of the source-side wind, light, and other uncertain resources are large and have a great influence on the stable operation of the system and the energy dispatch management. Therefore, the source-side wind forecasting is set to a higher weight; in addition, as the power grid plays a leading role in the integrated energy system by virtue of its perfect architecture and central hub advantages, correspondingly, the power load forecast is set to a higher weight.

This paper proposes three calculation indicators, namely, mean absolute percentage error (MAPE), mean accuracy 


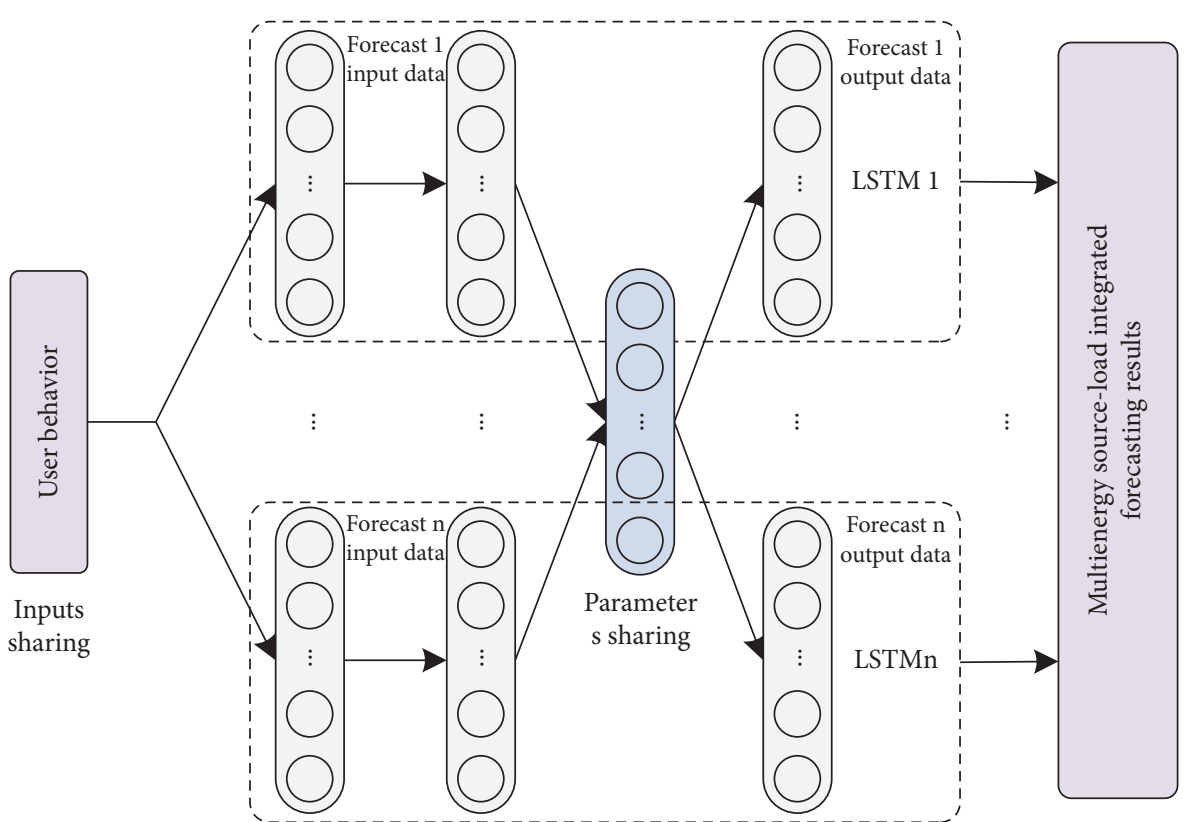

FIGURE 6: Interactive energy prediction model of RIES information based on parallel LSTM.

(MA), and weighted mean accuracy (WMA), as shown in the following equation:

$$
\begin{aligned}
\text { MAPE } & =\frac{1}{n} \sum_{i=1}^{n}\left|\frac{P(i)-\widehat{P}(i)}{P(i)}\right| \times 100 \%, \\
\mathrm{MA} & =1-\mathrm{MAPE}, \\
\mathrm{WMA} & =\lambda_{1} \mathrm{MA}_{1}+\lambda_{2} \mathrm{MA}_{2}+\cdots+\lambda_{k} \mathrm{MA}_{k} .
\end{aligned}
$$

In the equation, $P(i)$ and $\widehat{P}(i)$, respectively, represent the actual energy value and the predicted energy value at the first moment; $n$ is the sample data amount; $\mathrm{MA}_{k}$ represents the prediction accuracy of the $k$ prediction task; and $\lambda_{k}$ represents the weight coefficient of the $k$ prediction task. In this paper, the weight ratios of the energy predictions on both sides of the source and the load are set as shown in Table 1.

\section{Simulation Analysis}

6.1. Data Description. The experimental data in this paper are derived from the measured data provided by RIES in a certain area of Tianjin. The RIES consists of a power system, a thermal system, and a natural gas system. The source side includes energy supply equipment, such as wind turbines and photovoltaic power generation equipment. Coupling links include energy conversion equipment, such as cold and thermal power supply equipment, electric boilers, and gas boilers. Energy demand mainly includes power load, heat load, and cooling load. The load side and the energy storage side contain devices such as electrical energy storage and thermal energy storage to improve the flexibility of the system. In addition, in order to prove the validity of the source-load vertical information interaction, the historical data of the lighting load reflecting the typical electricity usage behavior of the user are also sampled. All historical energy data sampling interval is $1 \mathrm{~h}$, data from July 2015 to June 2016 are used as training set data, and data from July 2016 are used as test set data, with $1 \mathrm{~h}$ as time step; based on the historical data of the first 7 days, the energy supply and energy demand on both sides of the source and the load in the next day are predicted. The total 57168 samples were divided into two parts ( $92 \%$ as the train set and $8 \%$ as the test set). The forecasting models were trained by the train dataset and validated on the test dataset. The statistical information of the above dataset is shown in Table 2 .

6.2. Analysis of Examples. This section analyzes the effect of RIES multienergy information interactive energy prediction. The selected sunny day and rainy day of the test set were analyzed separately. The energy prediction results of wind power output, photovoltaic output, electric load, heat load, and cold load on both sides of the source and the load in the two scenarios are shown in Figures 7 and 8, respectively.

The prediction results of Figures 7 and 8 show that the proposed source-load parallel LSTM learning model can obtain ideal results in wind energy, photovoltaic, electric load, thermal load, and cold load energy prediction in sunny day scenario and rainy day scenario, which confirms the effectiveness of the model proposed in this paper. The prediction effect of each subtask in the parallel prediction results shows a large difference. Compared with the wind and light prediction on the source side, the predicted curves of the charge, heat, and cold load on the charge side are closer to the actual sequence curve, which is due to the intermittent and random nature of source-side wind power and photovoltaics.

Comparing Figures $7(\mathrm{c})-7(\mathrm{e})$, it can be found that the electric load is more volatile than the thermal load and the cold load, which is because the thermal inertia and cold 
TABLE 1: Weight settings of source-load double-sided energy prediction tasks.

\begin{tabular}{lccccc}
\hline Prediction task & Wind power & PV power & Electric load & Heat load & Cold load \\
\hline Weight coefficient & 0.25 & 0.25 & 0.2 & 0.15 & 0.15 \\
\hline
\end{tabular}

TABLE 2: The description of simulation dataset.

\begin{tabular}{lccccccc}
\hline Data type & Max $(\mathrm{kW})$ & Median $(\mathrm{kW})$ & Min $(\mathrm{kW})$ & Mean $(\mathrm{kW})$ & Std $(\mathrm{kW})$ & Train set size & Test set size \\
\hline Wind power & 317.561 & 233.774 & 36.612 & 216.102 & 78.964 & 8784 & 744 \\
Photovoltaic power & 235.000 & 0.000 & 0.000 & 42.507 & 70.557 & 8784 & 744 \\
Electric load & 696.585 & 584.923 & 209.872 & 523.803 & 139.174 & 8784 & 744 \\
Heat load & 148.473 & 92.719 & 60.532 & 100.076 & 27.240 & 8784 & 744 \\
Cold load & 436.964 & 223.213 & 39.046 & 221.949 & 135.304 & 8784 & 744 \\
Lighting load & 03.333 & 50.000 & 2.778 & 44.792 & 27.734 & 8784 & 744 \\
Multisource fusion & - & - & - & - & - & 52704 & 4464 \\
\hline
\end{tabular}

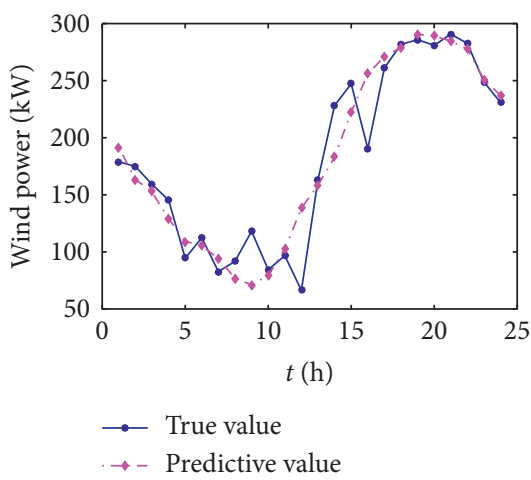

(a)

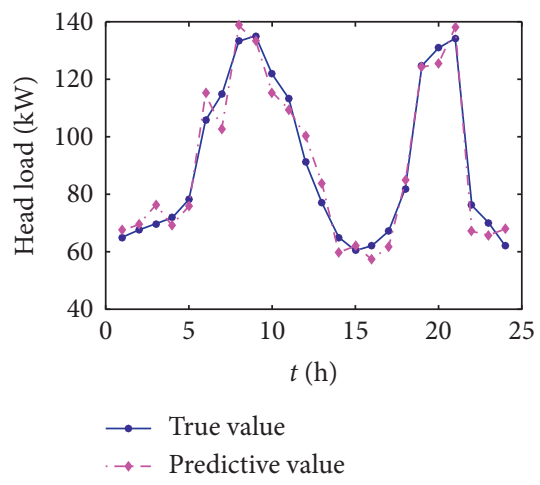

(d)

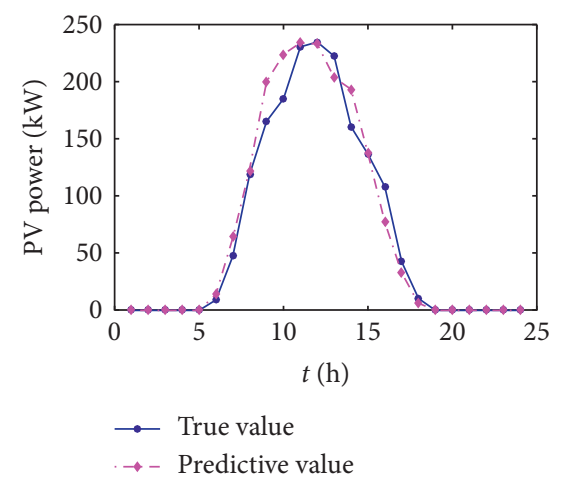

(b)

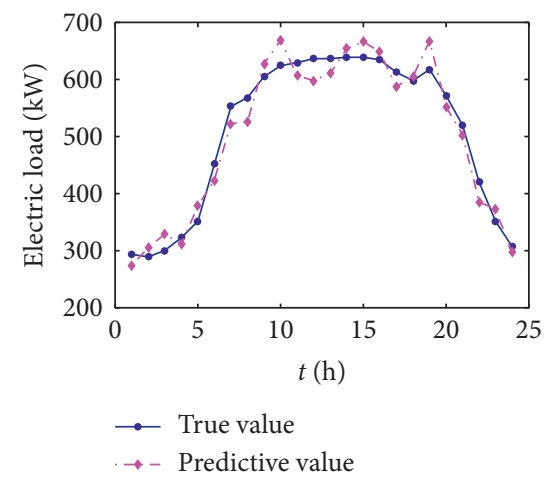

(c)

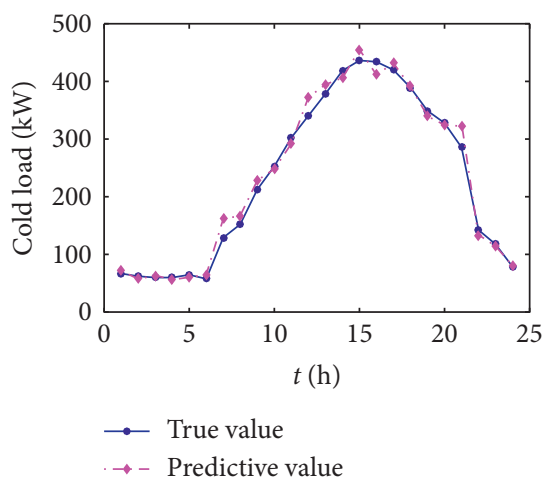

(e)

Figure 7: The results of source-load bilateral energy prediction within a sunny day. (a) Prediction of wind power. (b) Prediction of PV power. (c) Prediction of electric load. (d) Prediction of heat load. (e) Prediction of cold load.

inertia of the thermal system are stronger [39], and the volatility is also even smaller. Thermal inertia means that the dynamic change of the thermal system is a slow process from a time scale. Compared with the power system, the heat energy transmission exhibits a delay effect, and the upper and lower fluctuations of the heat load exhibit an inertial effect, and the cold inertia is similar. Because of the existence of thermal inertia and cold inertia, the thermal load in the thermal system is less random in the short-term predicted time scale, showing a characteristic of smooth variation, so its prediction uncertainty is also small, and the thermal load and the cold load are relative to the electricity. The prediction error of the load is also relatively small. Comparing Figures 7 and 8, it can be found that the uncertainty of actual photovoltaic power and actual wind power in rainy day scenes is larger than that of sunny days. The forecast curve of photovoltaic and wind power in sunny scenes is closer to the true value; the electric load and heat load in rainy scenes 


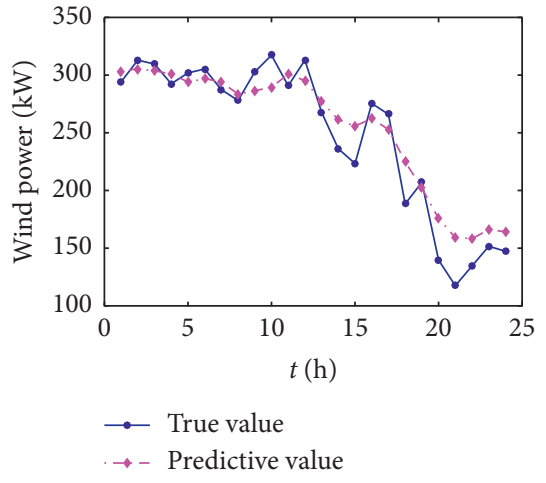

(a)

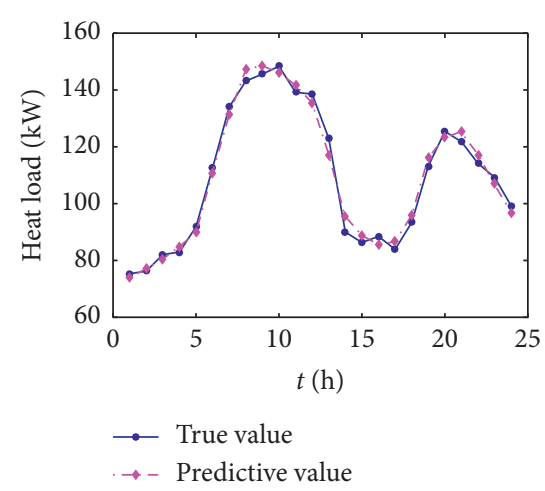

(d)

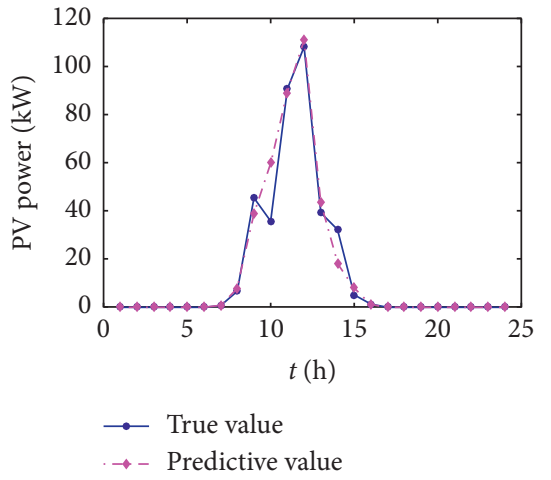

(b)

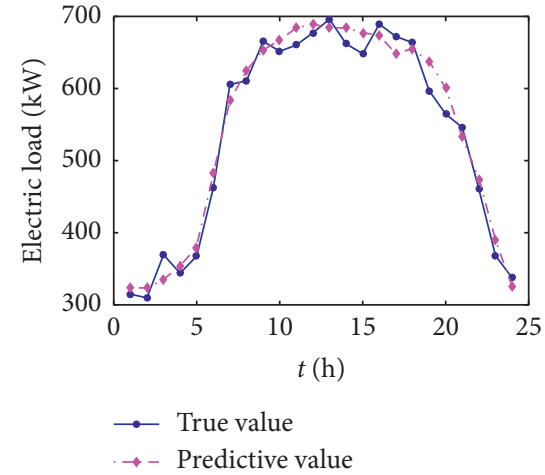

(c)

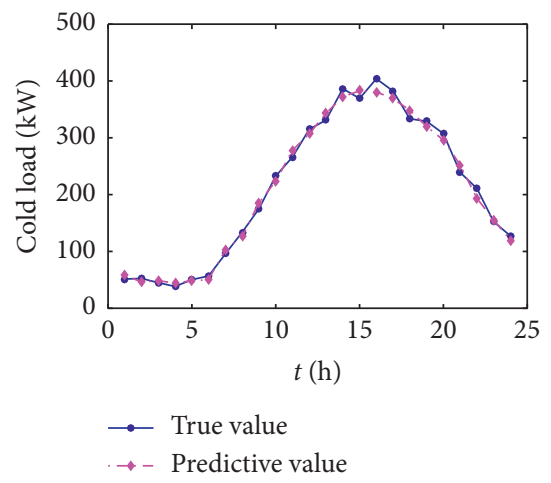

(e)

FIgURE 8: The results of source-load bilateral energy prediction within a rainy day. (a) Prediction of wind power. (b) Prediction of PV power. (c) Prediction of electric load. (d) Prediction of heat load. (e) Prediction of cold load.

should increase as a whole. This is due to the cold weather in the rainy scenes, which affects the user's energy use behavior and increases the user's demand for electricity and heat, thus increasing the electrical load and heat load.

\subsubsection{Comparative Analysis of Multienergy Load Information} Interaction. In order to verify the effect of interactive learning of horizontal and vertical multienergy information, the parallel LSTM predictions considering user energy behavior and parallel LSTM prediction without considering user energy behavior and the results of single LSTM prediction without considering user energy behavior are proposed to conduct a comparative analysis. In order to ensure the fairness of the experimental test, this paper uses the same dataset for training and testing.

The comparison experiment setup is shown in Table 3. In the parallel LSTM learning without considering the user's energy behavior, the input sample is based on the original prediction model $\mathrm{A}$, and the lighting load data reflecting the user behavior in the input sample are not considered. The prediction is based only on the historical data of the predicted object; the model $\mathrm{C}$ does not consider the single LSTM prediction of the user's energy behavior, and the input and output of each subprediction model only consider the input and output of a single energy, and there is also no parameter sharing between the LSTM unit of the different submodels. Model B uses parallel LSTM learning. There is parameter sharing among each subtask, which belongs to a multitask learning. Model C uses a single LSTM learning, and each subtask does not affect each other. It belongs to a single-task learning.

The prediction accuracy of the multienergy charge information interaction comparison experiment is shown in Table 4 and Figure 9. Through the analysis of Table 4 and Figure 9, it shows that the average prediction accuracy of the weights of the models A, B, and C are $0.9009,0.8917$, and 0.8728 , respectively. The prediction result of the model $\mathrm{A}$ is the best, the model B is the second, and the model C is the third. That is, considering the interaction of vertical and horizontal information, the energy predictions on both sides of the source and the load show better prediction accuracy.

Comparing the prediction results of Model B and Model C, for the five subprediction tasks, when the different quality energies are predicted together in parallel, a higher precision than the single prediction is obtained. It can be seen that parallel LSTM learning considering horizontal multienergy information interaction utilizes the coupled data between different systems to fully exploit the interactive information between different quality energy sources of the integrated energy system, so that the prediction performance of a certain energy can be improved. In addition, considering the multienergy horizontal information interaction, the predicted accuracy of the load-side electrical load, heat load, and 
TABLE 3: Contrast experiment setting of multisource source information interaction.

\begin{tabular}{lcc}
\hline Model & Vertical interaction & Horizontal interaction \\
\hline Model A & User energy behavior is considered & Parallel LSTM learning \\
Model B & User energy behavior is not considered & Parallel LSTM learning \\
Model C & User energy behavior is not considered & Single-LSTM learning \\
\hline
\end{tabular}

TABLE 4: Three kinds of model prediction results of information interaction comparison experiment.

\begin{tabular}{lcccccc}
\hline Precision & Wind power & PV power & Electric load & Heat load & Cold load & Mean precision \\
\hline Model A & 0.8602 & 0.8735 & 0.9236 & 0.9445 & 0.9407 & 0.9009 \\
Model B & 0.8542 & 0.8576 & 0.9113 & 0.9401 & 0.9368 & 0.8917 \\
Model C & 0.8414 & 0.8453 & 0.8836 & 0.9169 & 0.9124 & 0.8728 \\
\hline
\end{tabular}

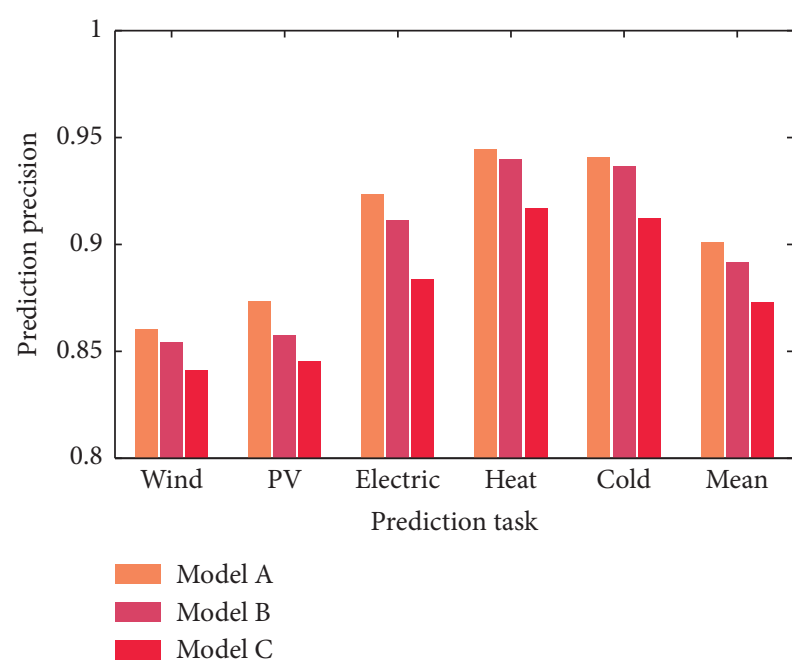

Figure 9: Comparison of prediction effects of three models.

cold load are increased by $3.13 \%, 2.53 \%$, and $2.67 \%$, respectively, while the predicted accuracy of the source-side wind power and photovoltaic is increased by $1.52 \%$ and $1.46 \%$ and the energy prediction accuracy of the charge side is relatively high. It can be seen that the source-side uncertainty resources are also affected by other energy interactions in the system, but the impact is small, while the charge side demand is largely affected by other energy interactions in the system. And the information interaction is more intimate between each other.

Comparing the prediction results of Model A and Model $B$, when the user's energy behavior information interacts between the source and the load in vertical direction, the average weight accuracy of the overall prediction is increased by $1.03 \%$. Comparing the performance improvement effects of the five subprediction tasks, it can be found that the percentage improvement of PV prediction accuracy is $1.85 \%$, and the improvement effect is relatively best, followed by the electrical load, while the prediction accuracy of wind power, heat load, and cooling load is not obvious. It can be seen that considering the source-load vertical information interaction can improve the energy prediction accuracy on both sides of the source and the load, but the specific user energy behavior has different differences in the energy prediction of different types of users, and the user lighting behavior is obviously related to photovoltaic power generation and user electricity. The interaction between loads is more tight, and its effect on PV prediction and electric load forecasting is more obvious.

6.2.2. Comparison of Deep Neural Network Algorithms with Other Algorithms. In order to verify the prediction effect of the LSTM deep neural network algorithm, the LSTM model and the autoregressive integrated moving average model (ARIMA) algorithm and the classic BP neural network (BPNN) algorithm in this paper are proposed. The simulation results of them were compared and analyzed. In order to ensure the fairness of the experimental test, this paper uses the same dataset for training and testing, and both use a single-task prediction strategy. The prediction results of the three algorithms are shown in Table 5 and Figure 10.

Observing the prediction results, the prediction effect of ARIMA algorithm is the most general, and the prediction accuracy of BPNN algorithm is much higher than that of ARIMA algorithm. The prediction accuracy of each task of LSTM deep learning prediction algorithm proposed in this paper is the highest. The ARIMA algorithm can not effectively use the historical information in the long-term sequence because it only uses a part of the historical information to train, which leads to the large prediction error. The LSTM algorithm can fully utilize its strong nonlinear learning ability and long-term and short-term memory advantages to exploit the information value of longterm historical data, so the grasp of the energy prediction law is more precise.

In addition, comparing the energy prediction results of the LSTM algorithm and the BPNN algorithm one by one, the LSTM algorithm predicts that the accuracy of the thermal load and the cold load prediction is more obvious. It can be seen that due to the greater inertia of the thermal system, the time scale of the change law is longer, which makes the prediction accuracy of the long-term and shortterm deep learning on the heat load and the cold load significantly higher than the electric load.

In order to evaluate the computational efficiency of algorithms, four forecasting scenarios are performed ten times so that the forecasting results can reliably be summarized as in Table 6. Parallel LSTM and Single LSTM have longer training time, but the prediction time is kept within 
TABLE 5: Comparison of prediction results of three algorithms.

\begin{tabular}{|c|c|c|c|c|c|c|}
\hline Precision & Wind power & PV power & Electric load & Heat load & Cold load & Mean precision \\
\hline LSTM & 0.8414 & 0.8453 & 0.8836 & 0.9169 & 0.9124 & 0.8728 \\
\hline BPNN & 0.8216 & 0.8314 & 0.8695 & 0.8912 & 0.8889 & 0.8542 \\
\hline ARMIA & 0.7733 & 0.7824 & 0.8282 & 0.8454 & 0.8408 & 0.8075 \\
\hline
\end{tabular}

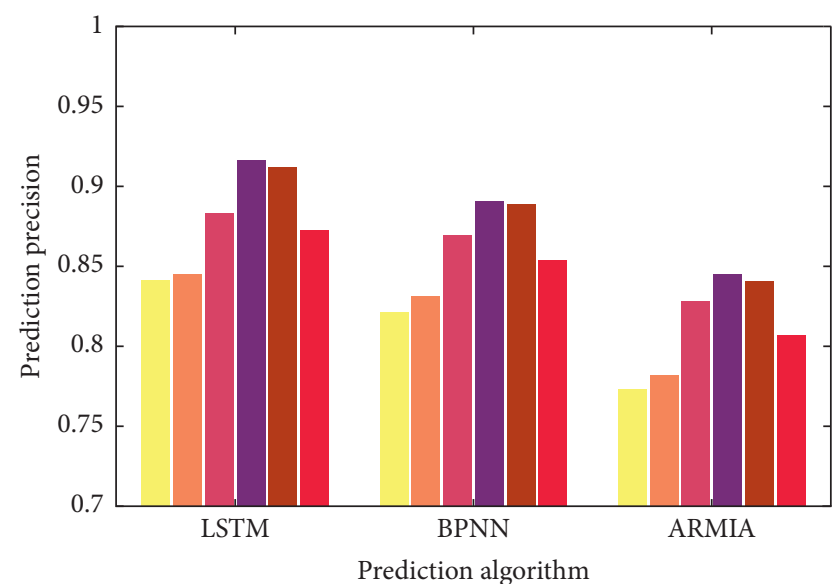

$\begin{array}{ll}\text { Prediction precision of } & \text { Prediction precision of } \\ \text { wind power } & \text { head load } \\ \text { Prediction precision of } & \text { Prediction precision of } \\ \text { PV power } & \text { cold load } \\ \begin{array}{l}\text { Prediction precision of } \\ \text { electric load }\end{array} & \begin{array}{l}\text { Weight average prediction } \\ \text { precision }\end{array}\end{array}$

Figure 10: Comparison of prediction effects of three algorithms.

TABle 6: Comparison of computational efficiency of four algorithms.

\begin{tabular}{lcc}
\hline Algorithm & $\begin{array}{c}\text { Training time } \\
\text { average }(\mathrm{sec})\end{array}$ & $\begin{array}{c}\text { Prediction time } \\
\text { average }(\mathrm{sec})\end{array}$ \\
\hline Parallel LSTM & 4363.74 & 10.02 \\
Single LSTM & 12183.37 & 36.62 \\
BPNN & 10949.11 & 22.53 \\
ARMIA & 546.96 & 2.15 \\
\hline
\end{tabular}

one minute and can meet the requirements of short-term forecasting. Further analysis shows that the parallel training time and prediction time of parallel LSTM is greatly improved compared to single LSTM due to the combination of five subtasks into one multitask learning. In summary, although the LSTM algorithm is time consuming in training time, for short-term prediction, the training process does not require online completion, and the prediction time of the LSTM algorithm, especially parallel LSTM, can fully meet the requirements of short-term prediction. Considering the prediction accuracy, the sacrifice of the LSTM algorithm in computing time is worthwhile.

\section{Conclusion}

In this paper, the horizontal and vertical information interaction characteristics between multiple energy loads in RIES are considered. Using LSTM network deep learning algorithm, a parallel LSTM-based multienergy-load information interactive short-term energy prediction method is proposed. Through the simulation of the test data, the following conclusions are drawn:

(1) The "information interaction" strategy proposed in this paper can effectively improve the prediction performance of source-load energy prediction in regional integrated energy systems

(2) Parallel prediction considering horizontal interaction mainly affects the prediction effect on the load side, while the information sharing of user illumination behavior considering vertical interaction mainly affects the improvement of PV prediction effect

(3) Compared with the conventional prediction algorithm, the LSTM deep learning algorithm can learn the deep hidden information of historical data, improve the prediction accuracy while meeting calculation time, and prove the effectiveness of the algorithm in the prediction of time series feature sequence

However, the performance of the proposed parallel LSTM algorithm may be further enhanced by some evolutionary algorithms or multiobjective optimization algorithms, such as differential evolution algorithm [40], multiobjective extremal optimization [41], and evolutionary multiobjective optimization algorithms [42-47]. Of course, some significant topics are worth researching on the optimization of the proposed parallel LSTM by multiobjective optimization.

\section{Data Availability}

The data used to support the findings of this study are available from the corresponding author upon request.

\section{Conflicts of Interest}

The authors declare no potential conflicts of interest.

\section{Acknowledgments}

This work was fully supported by the National Key Research and Development Program of China (no. 2018YFB0904200).

\section{References}

[1] A. Q. Huang, M. L. Crow, G. T. Heydt, J. P. Zheng, and S. J. Dale, "The future renewable electric energy delivery and management (FREEDM) system: the energy internet," Proceedings of the IEEE, vol. 99, no. 1, pp. 133-148, 2011.

[2] I. Colak, S. Sagiroglu, G. Fulli, M. Yesilbudak, and C. Covrig, "A survey on the critical issues in smart grid technologies," 
Renewable and Sustainable Energy Reviews, vol. 54, pp. 396405, 2016.

[3] C. Tu, X. He, Z. Shuai, and F. Jiang, "Big data issues in smart grid-a review," Renewable and Sustainable Energy Reviews, vol. 79, pp. 1099-1107, 2017.

[4] Y. Zhang, R. Yang, K. Zhang, H. Jiang, and J. J. Zhang, "Consumption behavior analytics-aided energy forecasting and dispatch," IEEE Intelligent Systems, vol. 32, no. 4, pp. 59-63, 2017.

[5] J. Deng, F. Wang, Y. Chen, and X. Zhao, "From industry 4.0 to energy 5.0: the concept, connotation and system framework of intelligent energy systems," Journal of Automation, vol. 41, no. 12, pp. 2003-2016, 2015.

[6] Q. Sun, L. Shi, Y. Ni, D. Si, and J. Zhu, "An enhanced cascading failure model integrating data mining technique," Protection and Control of Modern Power Systems, vol. 2, 2017.

[7] D. Zheng, A. T. Eseye, J. Zhang, and H. Li, "Short-term wind power forecasting using a double-stage hierarchical ANFIS approach for energy management in microgrids," Protection and Control of Modern Power Systems, vol. 2, pp. 1-10, 2017.

[8] Z. Li, L. Ye, Y. Zhao, X. Song, J. Teng, and J. Jin, "Short-term wind power prediction based on extreme learning machine with error correction," Protection and Control of Modern Power Systems, vol. 1, no. 1, pp. 1-8, 2016.

[9] H. Z. Wang, Z. X. Lei, X. Zhang, B. Zhou, and J. C. Peng, "A review of deep learning for renewable energy forecasting," Energy Conversion and Management, vol. 198, pp. 1-16, 2019.

[10] H. Z. Wang, G. B. Wang, G. Q. Li, J. C. Peng, and Y. T. Liu, "Deep belief network based deterministic and probabilistic wind speed forecasting approach," Applied Energy, vol. 182, pp. 80-93, 2016.

[11] M.-R. Chen, G.-Q. Zeng, K.-D. Lu, and J. Weng, “A two-layer nonlinear combination method for short-term wind speed prediction based on ELM, ENN, and LSTM," IEEE Internet of Things Journal, vol. 6, no. 4, pp. 6997-7010, 2019.

[12] H. Liu, X. Mi, and Y. Li, "Smart multi-step deep learning model for wind speed forecasting based on variational mode decomposition, singular spectrum analysis, LSTM network and ELM," Energy Conversion and Management, vol. 159, pp. 54-64, 2018.

[13] J. Chen, G.-Q. Zeng, W. Zhou, W. Du, and K.-D. Lu, "Wind speed forecasting using nonlinear-learning ensemble of deep learning time series prediction and extremal optimization," Energy Conversion and Management, vol. 165, pp. 681-695, 2018.

[14] W. Kong, Z. Y. Dong, Y. Jia, D. J. Hill, Y. Xu, and Y. Zhang, "Short-term residential load forecasting based on LSTM recurrent neural network," IEEE Transactions on Smart Grid, vol. 10, no. 1, 2017.

[15] H. Gao, W. Huang, X. Yang, Y. Duan, and Y. Yin, “Toward service selection for workflow reconfiguration: an interfacebased computing solution," Future Generation Computer Systems, vol. 87, pp. 298-311, 2018.

[16] L. Qi, Q. He, F. Chen et al., "Finding all you need: web APIs recommendation in web of things through keywords search," IEEE Transactions on Computational Social Systems, vol. 6, no. 5, 2019.

[17] L. Qi, R. Wang, C. Hu, S. Li, Q. He, and X. Xu, "Time-aware distributed service recommendation with privacy-preservation," Information Sciences, vol. 480, pp. 354-364, 2019.

[18] Y. Zhang and Q. Yang, "An overview of multi-task learning," National Science Review, vol. 5, no. 1, pp. 34-47, 2018.
[19] A. Argyriou, M. T. Evgeniou, and M. Pontil, "Convex multitask feature learning," Machine Learning, vol. 73, no. 3, pp. 243-272, 2008.

[20] T. Jebara, "Multitask sparsity via maximum entropy discrimination," Journal of Machine Learning Research, vol. 12, no. 1, pp. 75-110, 2011.

[21] L. Qi, Y. Chen, Y. Yuan, S. Fu, X. Zhang, and X. Xu, “A QoSaware virtual machine scheduling method for energy conservation in cloud-based cyber-physical systems," World Wide Web, vol. 5, pp. 1-23, 2019.

[22] R. Zhang, P. Xie, C. Wang, G. Liu, and S. Wan, "Classifying transportation mode and speed from trajectory data via deep multi-scale learning," Computer Networks, vol. 162, p. 106861, 2019.

[23] W. Wang, D. Wang, H. Jia et al., "Review of steady-state analysis of typical regional integrated energy system under the background of energy internet," Proceedings of the CSEE,12, pp. 3292-3306, 2016.

[24] S. Ding, S. Qu, Y. Xi, and S. Wan, "Stimulus-driven and concept-driven analysis for image caption generation," Neurocomputing, 2019.

[25] P. Vrba, V. Marik, P. Siano et al., "A review of agent and service-oriented concepts applied to intelligent energy systems," IEEE Transactions on Industrial Informatics, vol. 10, no. 3, pp. 1890-1903, 2014.

[26] W. Jia, C. Kang, and Q. Chen, "Analysis on demand-side interactive response capability for power system dispatch in a smart grid framework," Electric Power Systems Research, vol. 90, pp. 11-17, 2012.

[27] Z. C. Lipton, J. Berkowitz, and C. Elkan, "A critical review of recurrent neural networks for sequence learning," CoRR, 2015, https://arxiv.org/pdf/1506.00019.pdf.

[28] T. N. Sainath, O. Vinyals, A. Senior, and H. Sak, "Convolutional, long short-term memory, fully connected deep neural networks," in Proceedings of the 2015 IEEE International Conference on Acoustics, Speech and Signal Processing (ICASSP), pp. 4580-4584, IEEE, Brisbane, Australia, April 2015.

[29] Y. Guo, Z. Cheng, L. Nie, Y. Wang, J. Ma, and M. Kankanhalli, "Attentive long short-term preference modeling for personalized product search," ACM Transactions on Information Systems (TOIS), vol. 37, no. 2, p. 19, 2019.

[30] A. Graves, "Long short-term memory," Neural Computation, vol. 9, pp. 1735-1780, 1997.

[31] F. A. Gers, J. Schmidhuber, and F. Cummins, "Learning to forget: continual prediction with LSTM," Neural Computation, vol. 12, no. 10, pp. 2451-2471, 2000.

[32] M. L. Seltzer and J. Droppo, "Multi-task learning in deep neural networks for improved phoneme recognition," in Proceedings of the 2013 IEEE International Conference on Acoustics, Speech and Signal Processing, pp. 6965-6969, IEEE, Vancouver, Canada, May 2013.

[33] S. Wan, Y. Zhao, T. Wang, Z. Gu, Q. H. Abbasi, and K.-K. R. Choo, "Multi-dimensional data indexing and range query processing via Voronoi diagram for internet of things," Future Generation Computer Systems, vol. 91, pp. 382-391, 2019.

[34] Z. Gao, D. Y. Wang, S. H. Wan, H. Zhang, and Y. L. Wang, "Cognitive-inspired class-statistic matching with triple-constrain for camera free 3D object retrieval," Future Generation Computer Systems, vol. 94, pp. 641-653, 2019.

[35] C. A. Kang, A. R. Brandt, and L. J. Durlofsky, "Optimal operation of an integrated energy system including fossil fuel 
power generation, $\mathrm{CO} 2$ capture and wind," Energy, vol. 36, no. 12, pp. 6806-6820, 2011.

[36] Q. Zhang, S. Wan, B. Wang, D. W. Gao, and H. Ma, "Anomaly detection based on random matrix theory for industrial power systems," Journal of Systems Architecture, vol. 95, pp. 67-74, 2019.

[37] Z. Gao, H. Z. Xuan, H. Zhang, S. Wan, and K. K. R. Choo, "Adaptive fusion and category-level dictionary learning model for multi-view human action recognition," IEEE Internet of Things Journal, p. 1, 2019.

[38] W. Wu, K. Chen, Y. Qiao, and Z. Lu, "Probabilistic short-term wind power forecasting based on deep neural networks," in Proceedings of the 2016 International Conference on Probabilistic Methods Applied to Power Systems (PMAPS), pp. 1-8, Beijing, China, October 2016.

[39] K. M. Powell, A. Sriprasad, W. J. Cole, and T. F. Edgar, "Heating, cooling, and electrical load forecasting for a largescale district energy system," Energy, vol. 74, pp. 877-885, 2014.

[40] A. Peimankar, S. J. Weddell, T. Jalal, and A. C. Lapthorn, "Multi-objective ensemble forecasting with an application to power transformers," Applied Soft Computing, vol. 68, pp. 233-248, 2018.

[41] G.-Q. Zeng, J. Chen, L.-M. Li et al., “An improved multiobjective population-based extremal optimization algorithm with polynomial mutation," Information Sciences, vol. 330, pp. 49-73, 2016.

[42] Y.-L. Hu and L. Chen, "A nonlinear hybrid wind speed forecasting model using LSTM network, hysteretic ELM and Differential Evolution algorithm," Energy Conversion and Management, vol. 173, pp. 123-142, 2018.

[43] G.-Q. Zeng, J. Chen, Y.-X. Dai, L.-M. Li, C.-W. Zheng, and M.-R. Chen, "An improved Design of fractional order PID controller for automatic regulator voltage system based on multi-objective extremal optimization," Neurocomputing, vol. 160, pp. 173-184, 2015.

[44] X. Xu, R. Gu, F. Dai, L. Qi, and S. Wan, "Multi-objective computation offloading for internet of vehicles in cloud-edge computing," Wireless Networks, pp. 1-19, 2019.

[45] S. Wan, X. Li, Y. Xue, W. Lin, and X. Xu, "Efficient computation offloading for internet of vehicles in edge computing-assisted 5G networks," The Journal of Supercomputing, pp. 1-30, 2019.

[46] B. Wang, S. Wan, X. Zhang, and K.-K. R. Choo, "A novel index for assessing the robustness of integrated electrical network and a natural gas network," IEEE Access, vol. 6, pp. 40400-40410, 2018.

[47] Y. Yin, F. Yu, Y. Xu, L. Yu, and J. Mu, "Network locationaware service recommendation with random walk in cyberphysical systems," Sensors, vol. 17, no. 9, p. 2059, 2017. 


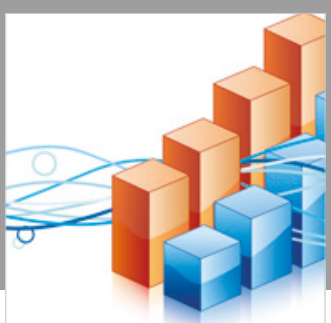

Advances in

Operations Research

\section{-n-m}
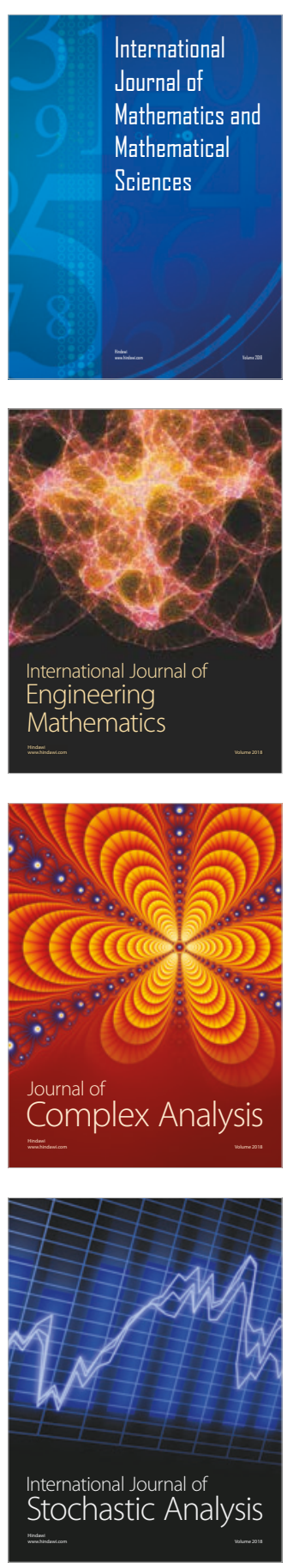
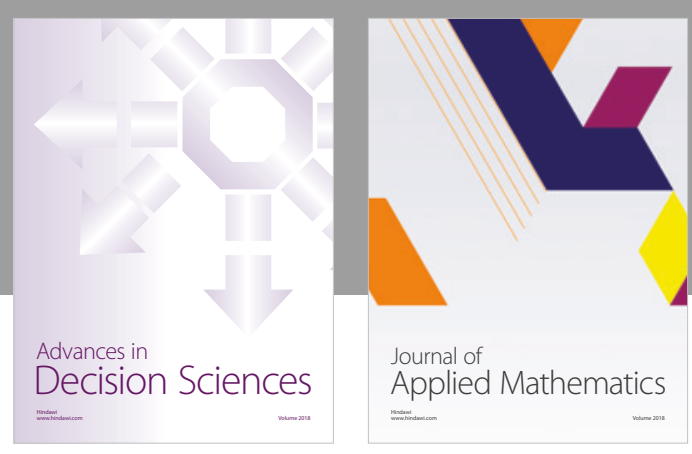

Journal of

Applied Mathematics
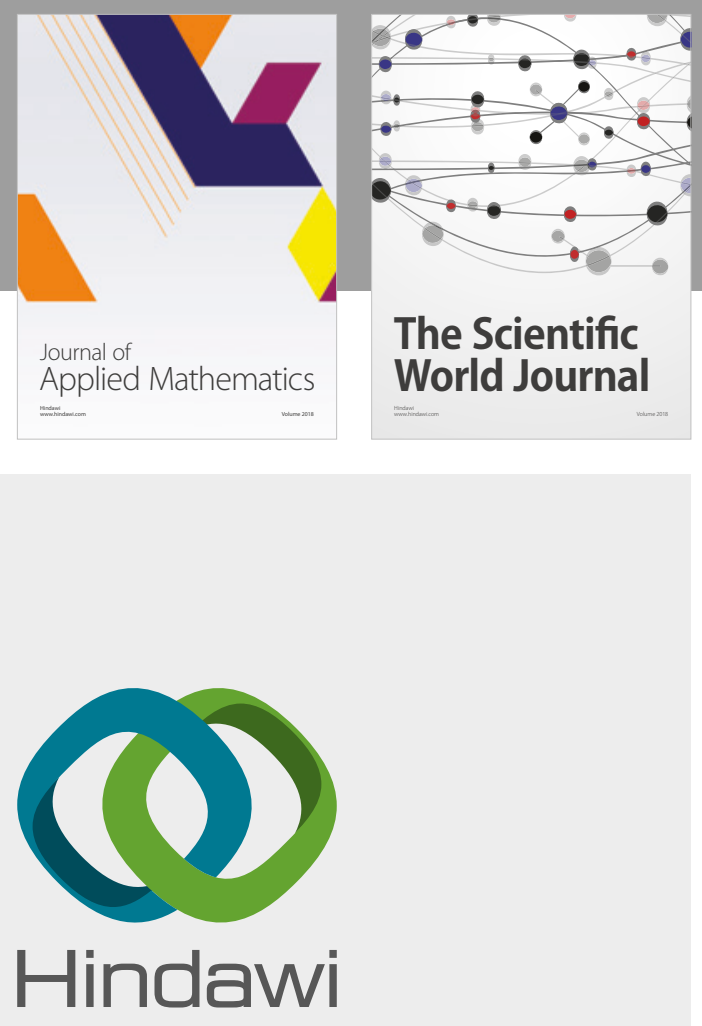

Submit your manuscripts at

www.hindawi.com

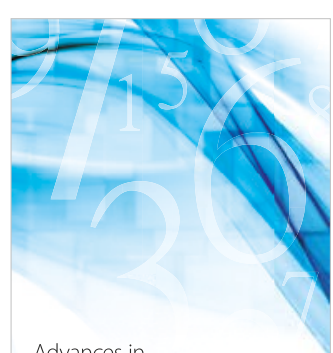

Advances in
Numerical Analysis
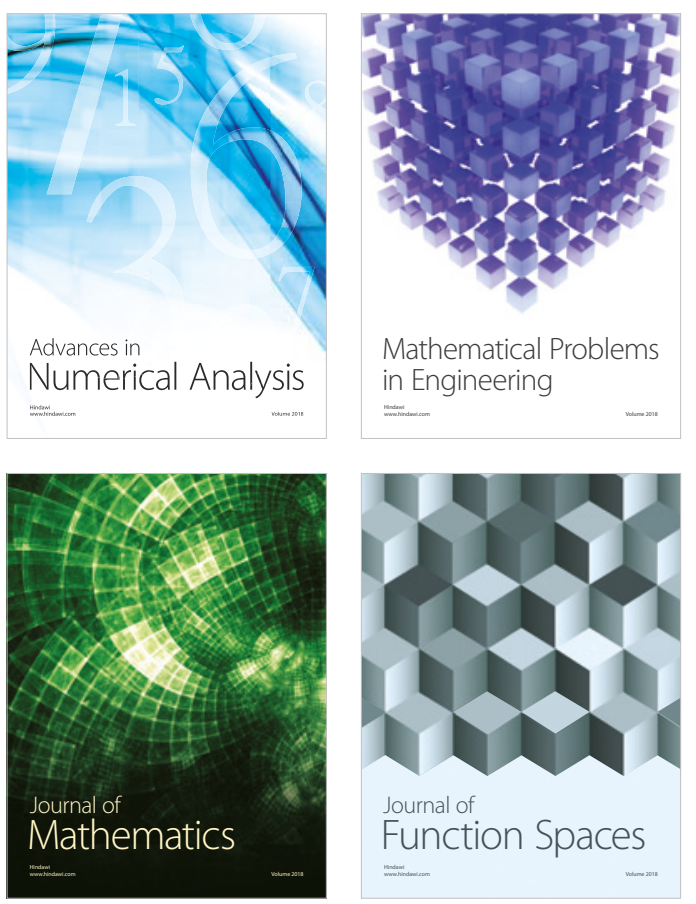

Mathematical Problems in Engineering

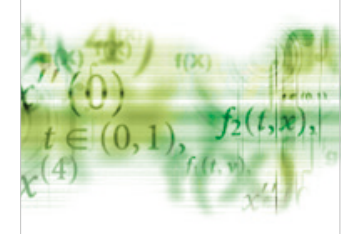

International Journal of

Differential Equations

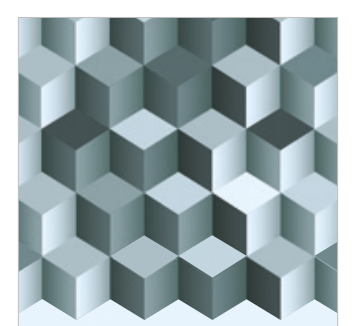

Journal of

Function Spaces
The Scientific

World Journal

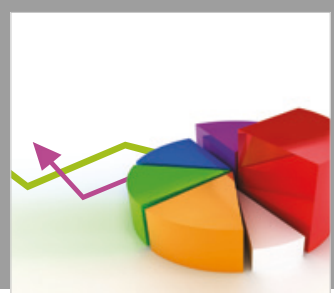

Journal of

Probability and Statistics
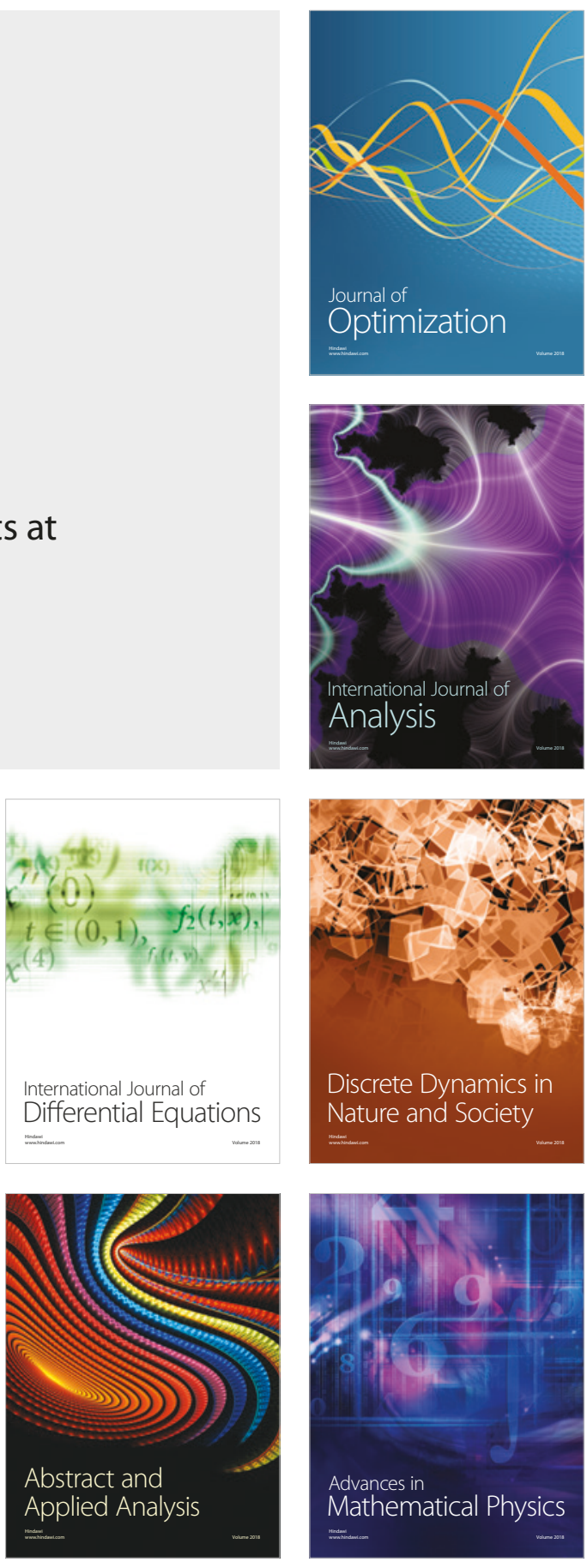